\title{
Relationship between rescue distance and the quality of simulated CPR: a pilot study with lifeguards
}

\author{
Brais Ruibal-Lista1,2, J. Enrique Moral-García1,3, Sergio López-García ${ }^{1,2,3, \text { * }}$
}

\author{
${ }^{1}$ Faculty of Education, Pontifical \\ University of Salamanca, Salamanca \\ Spain \\ ${ }^{2}$ Grupo de Investigación en Actividades \\ de Prevención y Socorrismo (GIAPS). \\ Universidade da Coruña (UDC), Spain \\ ${ }^{3}$ Grupo de Investigación en Actividad \\ Física, Deporte y Salud (GIADES). \\ Pontifical University of Salamanca, Spain

\section{*Correspondence \\ slopezga@upsa.es} \\ (Sergio López-García)
}

\begin{abstract}
Objective: The aim of this research was to investigate if the distance travelled in a 'rescue' is associated with differences in the quality of the CPR provided.

Methods: A group of 10 lifeguards performed simulated CPR for 2 minutes at rest. Next, they performed 2 rescues, one of 50 meters and the other of 100 meters. After each rescue, the lifeguards immediately performed simulated CPR.

Results: The time invested in the rescue of 50 meters was significantly lower than in the 100 meters $(P<0.001)$. Simulated CPR at rest obtained high quality values in the compressions $(94.8 \pm 9.6 \%)$ but not in the ventilations $(41.0 \pm 22.8 \%) .50$ and 100 meter rescues were associated with a significant decline in the overall quality of CPR $(68.4 \pm 11.0$ vs. $51.0 \pm 9.3$ vs. $49.7 \pm 7.2 \%, P=0.002)$, correctness of hand position $(100 \%$ vs. $91.0 \pm 7.0$ vs. $85.9 \pm 12.3, P=0.006)$, and in the quality of the ventilations $(41.0 \pm 22.8$ vs. $12.0 \pm 17.5$ vs. $11.0 \pm 12.8 \%, P=0.001)$. The quality of the above measures was similar in 50 and 100 meter rescues.

Conclusions: Rescues of 50 and 100 meters were similarly associated with a decrease in the quality of simulated CPR vs. at rest simulated CPR. Lifeguards should practice performing CPR following rescue activities with added focus on performing rescue ventilations correctly.
\end{abstract}

\section{Keywords}

Cardiopulmonary-resuscitation; Drowning; Emergency; Lifeguard; Rescue

\section{Introduction}

Drowning currently causes around 370,000 deaths a year worldwide [1]. The role of the lifeguard is to try to avoid this type of accident taking place [2]. When a lifeguard reaches a person who has suffered water inhalation, the lifeguard must perform the first rescue breaths in the water [3] and immediately complete the rescue as soon as possible and initiate CPR on the mainland [4].

The performance of an rescue attempt causes a high physiological strain in the lifeguard [5, 6], including tachycardia, hyperventilation and the accumulation of lactate [7-9]. Despite this physiological stress, the lifeguard also needs to be able to provide high quality chest compressions and ventilations, in order to provide adequate CPR $[10,11]$.

Specific materials have been shown to reduce rescue time [12], however, these material do not prevent a significant decrease in the quality of compressions [13] and ventilations [14], caused by the physiological stress incurred during the rescue. Also, evidence shows that lifeguards have a limited awareness of their actual ability to perform CPR and consequently make mistakes when they carry out CPR protocols [15]. Finally, it is important to indicate that the distance at which people require aquatic rescue varies significantly [16].
In this study we wanted to investigate if the distance covered during an aquatic rescue is associated with differences in the quality of the CPR provided by lifeguards.

\section{Methods}

\subsection{Study population}

Ten professional lifeguards registered in the official registry of aquatic lifeguards of Galicia (Spain) participated in the study. The selected lifeguards had recently completed their mandatory training to be a professional lifeguard according to Spanish law.

A quasi-experimental design was used to evaluate the rescue process, the lifeguards physiological variables and the overall success of the Cardiopulmonary Resuscitation protocol (Fig. 1). All participants signed an informed consent form agreeing to the use and publication of the data obtained.

The study was approved by the Ethics and Research Committee of the Universidade da Coruña (CEI-UDC). The research was performed in accordance to the Declaration of Helsinki of 1975 . 

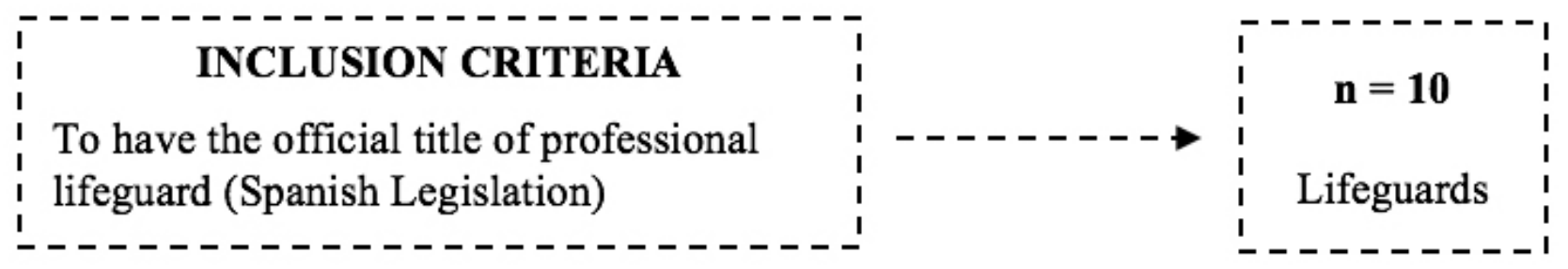

\begin{tabular}{|l|l|}
\hline Day 1 & Day 2 \\
\hline
\end{tabular}

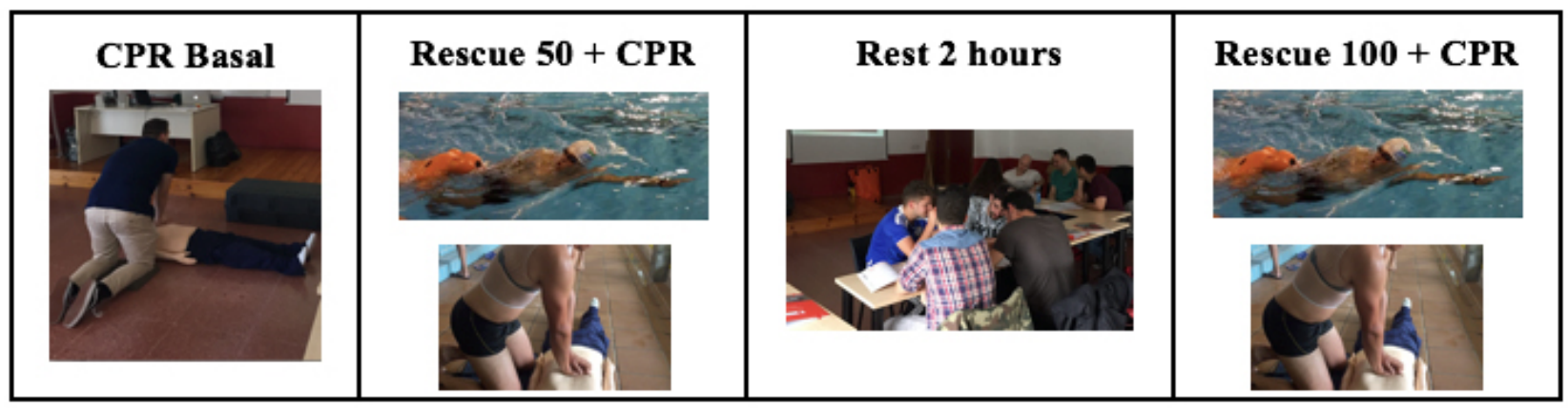

Rescue: FT: Time invested to put the Fins; AT: Approach Time; TT: Towing Time; TotalT: Total Time; Rescue50: Rescue of 50 meters; Rescue 100: Rescue of $100 \mathrm{~m}$.

CPR: QCPR: Quality CPR; CCC: Correct Chest Compressions; CCR: Compressions with Correct Rhythm; CCD: Compressions with Correct Depth; CHP: Compressions with Correct Hand Position; QV: Quality Ventilation; TV: Tidal Volume.

Physiological Variables: HR: Heart Rate; RPE: Rating of Perceived Exertion.

F I G U R E 1. Flow chart of the experimental design.

\subsection{Rescue tests}

At first, all the rescuers performed a simulated CPR test (30 : 2) for 2 minutes. The maximum heart rate of each participant was calculated following the equation of Tanaka [17], designed for active adults.

The following day, all rescuers performed 2 pool rescues, one of 50 meters (Rescue 50 ) and the other of 100 (Rescue $_{100}$ ). In both rescues, the lifeguard had to put on the swimming fins, enter the water, swim 25 or 50 meters of approach through the front crawl swim and 25 or 50 meters of towing swim keeping the victim's airways above the water. All participants performed the 50-meter rescue first and then the 100-meter rescue. Between both rescues, all participants rested for 2 hours to ensure complete recovery [7]. In both rescues, the time invested to put the fins (FT), the approach time (AT), the towing time (TT) and the total time (TotalT) were measured.

The heart rate (HR) measurement was carried out with a Sunnto $\AA$ pulsometer with a strap placed on the chest that sent the data recorded in real time to a computer. The values achieved at the end of each rescue and at the beginning and end of each simulated CPR test were selected. A modified Borg Scale (from 1 to 10) was used to measure the rating of perceived exertion (RPE). Each lifeguard had to point a finger at the level of fatigue after each rescue and each simulated CPR sequence.

A mannequin with the same characteristics as the one used in competitions regulated by the International Lifeguard Federation, was used. This mannequin was used also in other studies as a victim $[18,19]$. The rescues were carried out with fins (Cressi Clio, Cressi-Sub C, Italy).

All the rescuers were informed that the test simulated a real drowning situation and they had to exert themselves physically and technically according to the demands of the situation.

\subsection{Simulated CPR tests}

At the end of each of the rescues, lifeguards immediately performed a 2-minute simulated CPR test. During all simulated $\mathrm{CPR}$ tests, the following parameters were recorded: the general quality (QCPR), the percentage of correct compressions (QCC), the percentage of compressions with adequate depth (QDC), the position of the hands (HP), the decompression of the thorax (CD), the percentage of correct ventilations (QV) and the mean volume of air insufflated (TV).

The CPR quality was analyzed with the Resusci Anne ${ }^{\circledR}$ SkilReporterTM connected to a laptop with the Laerdal PC Skill Reporter System Program software for Windows (Laerdal Medical Corporation, Stavanger, Norway). Compressions with a depth of 5-6 centimeters, with correct hand position, 


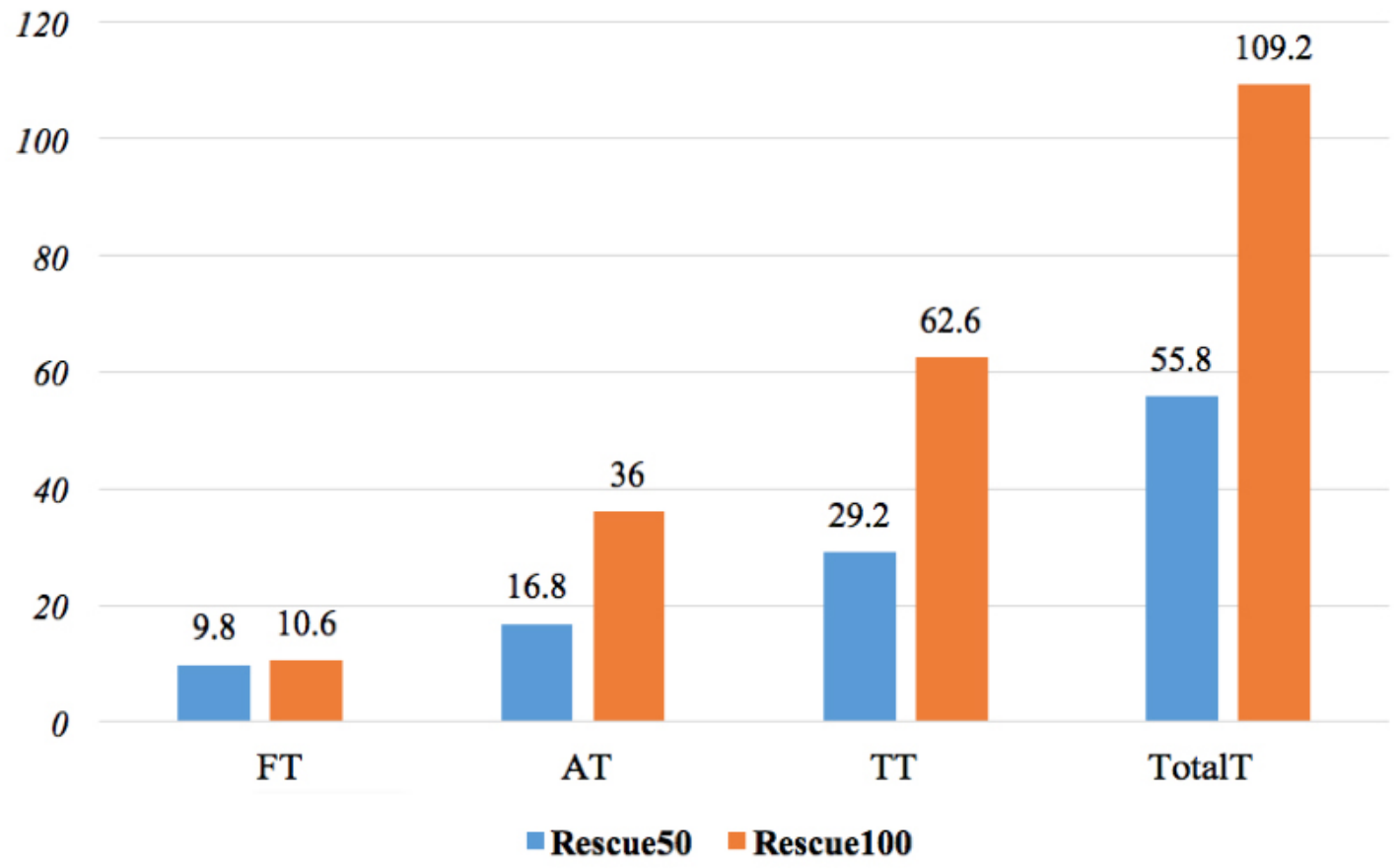

F I G URE 2. Rescue $_{50}$ and Rescue 100 results. FT, Time to put the fins; AT, Aproach Time; TT, Towing Time; TotalT, Total Time.

complete re-expansion of the chest, and ventilations with 500$600 \mathrm{~mL}$ of insufflated air were recorded as correct. CPR quality was calculated as a percentage (\%) using the following equation: $\mathrm{QCPR}=[(\mathrm{QCC}+\mathrm{QV}) / 2]$, as previously used in other studies [20].

\subsection{Statistics}

The variables were expressed by measures of central tendency and dispersion (mean and standard deviation). The results of the tests were analyzed using statistical software (SPSS, version 22.0, SPSS Inc.). Normality was verified by the Shapiro-Wilk test.

For the comparisons of the temporary variables between Rescue $_{50}$ and Rescue 100 , paired sample $t$-tests or the Wilcoxon Signed Rank Test was applied, and for comparisons between the simulated CPR results, ANOVA with repeated measures was applied. A level of significance was established at $P<$ 0.05 .

\section{Results}

The total sample was 10 male lifeguards. The mean age was $22.9 \pm 2.4$ years, the weight $78.3 \pm 9.7 \mathrm{~kg}$, the height 174.37 $\pm 8.0 \mathrm{~cm}$ and the BMI $25.7 \pm 2.9 \mathrm{~kg} / \mathrm{m}^{2}$. The theoretical maximum heart rate was $191 \pm 2$ beats per minute [17].

\subsection{Rescue results}

The time spent in fitting the fins was similar in both cases (TF: $9.8 \pm 2.5$ s. vs. $10.6 \pm 2.3 \mathrm{~s} ; P=0.443$ ), although the rest of the temporal parameters were lower in the Rescue 50 group (AT: $16.8 \pm 3.7$ s. vs. $36.0 \pm 5.1 \mathrm{~s} ; P<0.001$; TT: $29.2 \pm 4.0$ s. vs. $62.5 \pm 5.5 \mathrm{~s} ; P<0.001$; TotalT: $55.8 \pm 8.4$ s. vs. 109.2 $\pm 10.1 \mathrm{~s} ; P<0.001)$. All results are shown in Fig. 2.

\subsection{Simulated CPR results}

The results of the baseline simulated CPR test $\left(\mathrm{CPR}_{\mathrm{BASELINE}}\right)$ were of good/high quality standard [21]. A high effectiveness in the percentage of correct compressions (QCC $=94.5 \pm$ $5.7 \%$ ) was observed, due to a high effectiveness of the compressions adequate depth $(\mathrm{CCP}=88.7 \pm 13.2 \%)$ and with a maximum effectiveness in the hand position $(\mathrm{PM}=100 \%)$.

However, although the mean volume of insufflated air was maintained between the recommended values (VT: $540 \pm 87$ $\mathrm{mL}$ ), the percentage of correct ventilations did not reach $50 \%$ effectiveness threshold (QV $=45.0 \pm 21.6 \%$ ) (Fig. 3).

In Table 1 it can be observed that the comparison between the three CPR tests: Baseline $\left(\mathrm{CPR}_{\mathrm{BASELINE}}\right)$; post 50 metersrescue $\left(\mathrm{CPR}_{50}\right)$ and post 100 meters-rescue $\left(\mathrm{CPR}_{100}\right)$. Significant differences were found in the overall quality of the CPR at $\mathrm{CPR}_{\text {BASELINE }}, \mathrm{CPR}_{50}$ and $\mathrm{CPR}_{100}$ respectively (QCPR: $69.8 \pm$ 11.4 vs. $47.2 \pm 16.4$ vs. $46.5 \pm 14.4 \% ; P=0.010)$, the correct chest compressions (QCC: $94.5 \pm 5.7 \%$ vs. $78.8 \pm 16.8 \%$ vs. $77.6 \pm 13.9 \% ; P=0.004)$, the total number of compressions (TCC: $134 \pm 20$ vs. $151 \pm 11$ vs. $154 \pm 10 ; P=0.001$ ), the compressions with adequate rhythm (CCR: $89.5 \pm 11.1 \%$ vs. $48.0 \pm 46.1 \%$ vs. $39.0 \pm 39.5 \% ; P=0.005)$, the hands position (HP: $100 \%$ vs. $94.8 \pm 7.3$ vs. $92.0 \pm 8.5 ; P=0.018$ ), the quality of the ventilations (QV: $45.0 \pm 21.6 \mathrm{vs} .15 .5 \pm$ 32.7 vs. $14.2 \pm 21.6 \% ; P=0.021$ ) and the tidal volume (VT: $540 \pm 87$ vs. $709 \pm 83$ vs. $736 \pm 96 \mathrm{~mL} ; P<0.001)$.

There were significant differences between the $\mathrm{CPR}_{\mathrm{BASELINE}}$ and the $\mathrm{CPR}_{50}$ as well as between the $\mathrm{CPR}_{\mathrm{BASELINE}}$ and the $\mathrm{CPR}_{100}$. There were significant differences between $\mathrm{CPR}_{50}$ and $\mathrm{CPR}_{100}$ only in two parameters, CCR $(48.0 \pm 46.1 \%$ vs. $39.0 \pm 39.5 \% ; P=$ $0.022)$ and CHP $(94.8 \pm 7.3 \%$ vs. $92.0 \pm 8.5 \% ; P=0.044)$ (Fig. 4). 


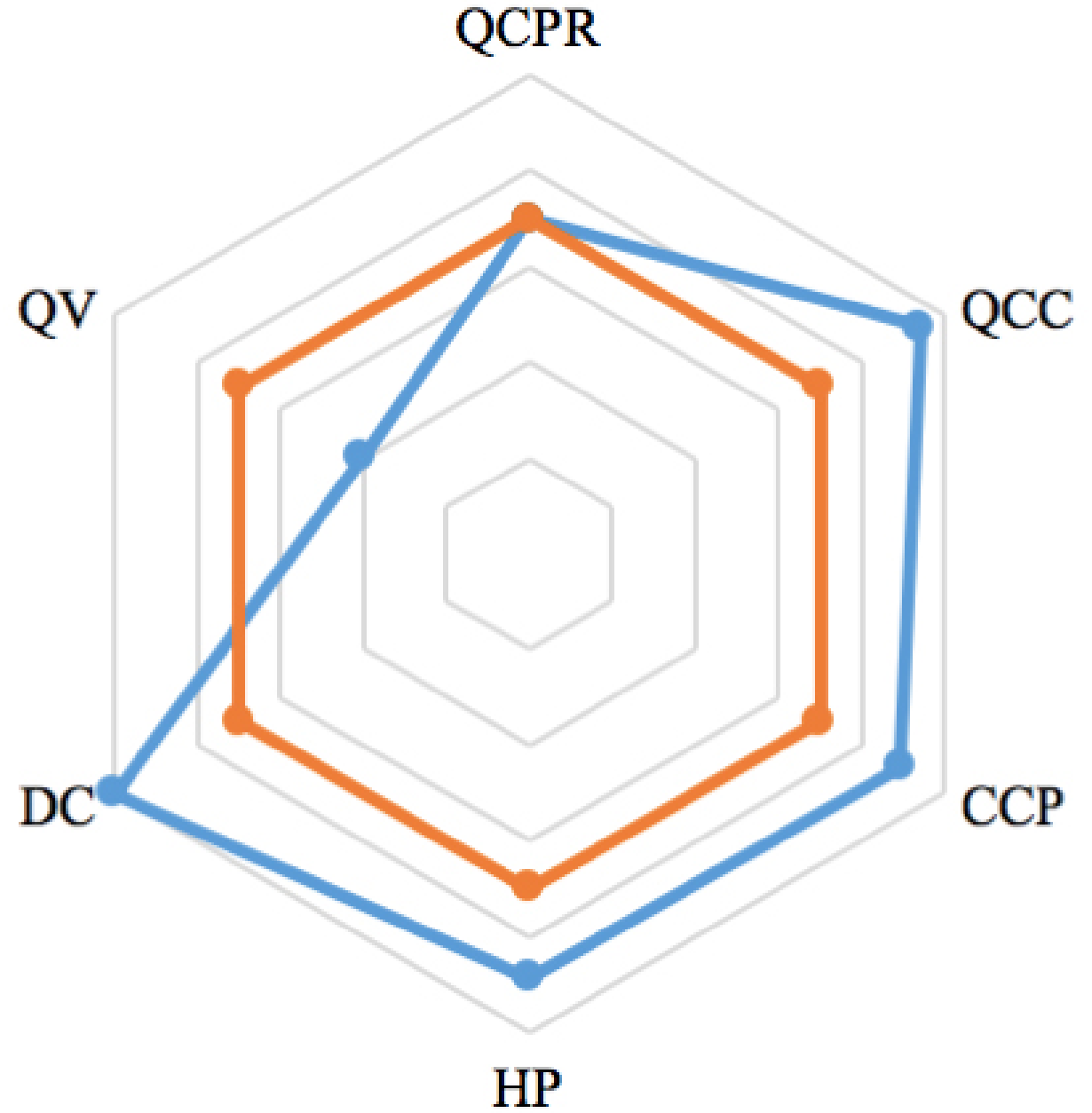

- CPRBasal CPRQuality

F I G U RE 3. Comparative between CPR ${ }_{\text {BASAL }}$ and CPR $R_{\text {QUALITY }}$

TA B L E 1. Simulated CPR test results: CPR BASELINE, post-rescue (50 meters) and post-rescue (100 meters)

\begin{tabular}{|c|c|c|c|c|c|c|c|}
\hline \multirow[b]{2}{*}{ Variables } & \multirow[b]{2}{*}{$\mathrm{CPR}_{\mathrm{BASAL}}$} & \multirow[b]{2}{*}{$\mathrm{RCP}_{50}$} & \multirow[b]{2}{*}{$\mathrm{RCP}_{100}$} & \multirow[b]{2}{*}{$P$-value } & \multicolumn{3}{|c|}{ Comparación por pares } \\
\hline & & & & & Baseline-Rescue $_{50}$ & Baseline-Rescue $_{100}$ & Rescue $_{50}-$ Rescue $_{100}$ \\
\hline QCPR (\%) & $69.8 \pm 11.4$ & $47.2 \pm 16.4$ & $46.5 \pm 14.4$ & $0.010^{a}$ & 0.044 & 0.034 & 0.986 \\
\hline $\mathrm{CCC}(\%)$ & $94.5 \pm 5.7$ & $78.8 \pm 16.8$ & $77.6 \pm 13.9$ & $0.004^{a}$ & 0.043 & 0.013 & 1.000 \\
\hline TCC (comp) & $106 \pm 2$ & $119 \pm 2$ & $120 \pm 2$ & $0.001^{a}$ & 0.003 & 0.003 & 1.000 \\
\hline CCR $(\%)$ & $89.5 \pm 11.1$ & $48.0 \pm 46.1$ & $39.0 \pm 39.5$ & $0.005^{b}$ & 0.281 & 0.022 & 0.943 \\
\hline CCD $(\%)$ & $88.7 \pm 13.2$ & $75.5 \pm 23.6$ & $79.5 \pm 22.5$ & $0.682^{b}$ & - & - & - \\
\hline CHP (\%) & 100 & $94.8 \pm 7.3$ & $92.0 \pm 8.5$ & $0.018^{b}$ & 0.094 & 0.044 & 0.737 \\
\hline QV (\%) & $45.0 \pm 21.6$ & $15.5 \pm 32.7$ & $14.2 \pm 21.6$ & $0.021^{b}$ & 0.034 & 0.025 & 0.911 \\
\hline $\mathrm{TV}(\mathrm{mL})$ & $540 \pm 87$ & $709 \pm 83$ & $736 \pm 96$ & $<0.001^{a}$ & $<0.001$ & $<0.001$ & 0.510 \\
\hline
\end{tabular}

CCC, Correct Chest Compressions; CCD, Compressions with Correct Depth; CCR, Compressions with Correct Rhythm; CHP, Compressions with Correct Hand Position; QCPR, Quality CPR [QCC $+Q V) / 2] ; Q V$, Ventilation Quality; TCC, Total Chest Compresssions; TV, Tidal Volume.

${ }^{a}$ : Friedman Statistic; ${ }^{b}$ : ANOVA test of repeated measures.

Table 2 shows the results of the HR and the rating of perceived exertion (RPE) after the efforts made in the rescue and simulated CPR. It was found that HR and RPE values were lower in the 50-meter rescue compared to the 100-meter rescue
(HR: $164 \pm 7$ vs. $174 \pm 7, P=0.002$, RPE: $7.3 \pm 0.6$ vs. 8.3 $\pm 0.6, P=0.011)$. However, the values of HR and RPE did not show significant differences after completing the simulated $\mathrm{CPR}$, in both the 50 meter and 100 meter rescues (HR: $128 \pm$ 


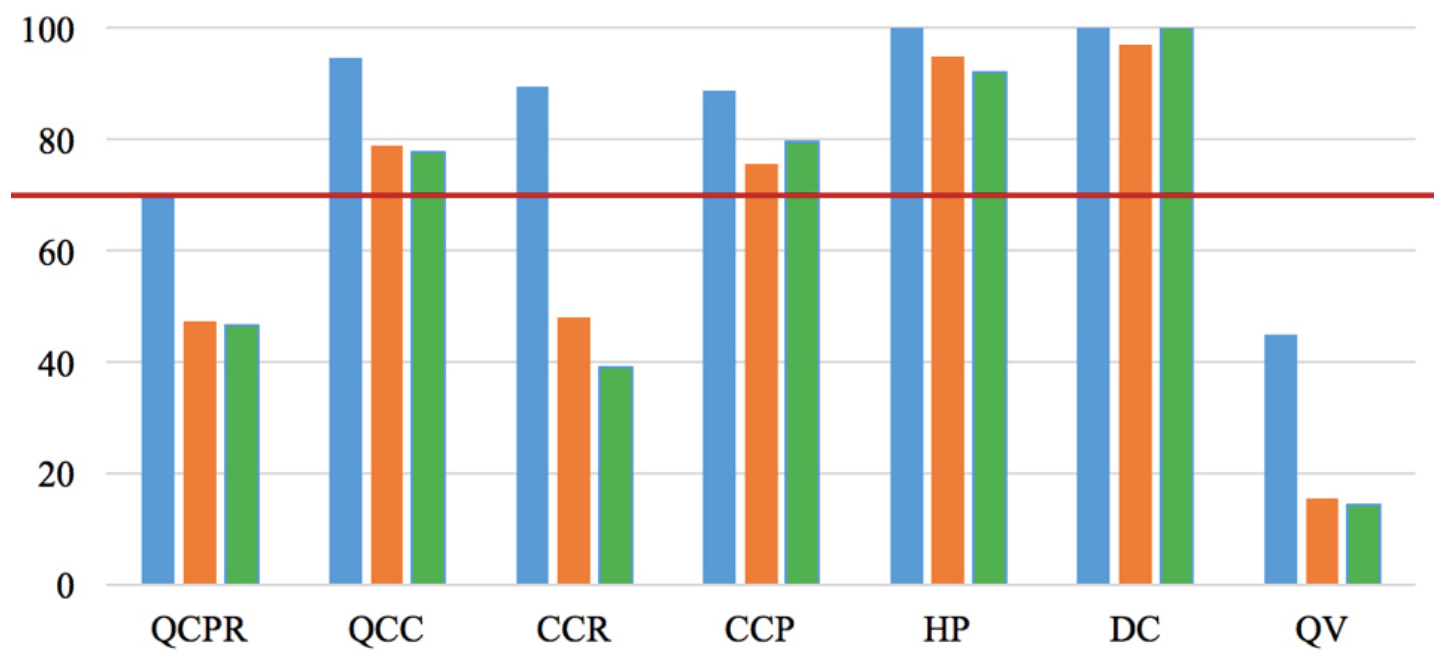

\section{-CPRBasal $\square$ CPR50 $\square$ CPR100}

F I G U R E 4. Comparative between $\mathrm{CPR}_{\mathrm{BASAL}}, \mathrm{CPR}_{50}$ and $\mathrm{CPR} \mathrm{R}_{100}$.

TA B LE 2. Differences between HR and RPE of the Rescues and the CPR tests

\begin{tabular}{ccccc} 
& & 50 meters & 100 meters & $P$-value \\
Rescue & HR & $164 \pm 7$ & $174 \pm 7$ & $0.002^{a}$ \\
& RPE & $7.3 \pm 0.6$ & $8.3 \pm 0.6$ & $0.011^{b}$ \\
CPR & HR & $128 \pm 7$ & $130 \pm 6$ & $0.149^{c}$ \\
& RPE & $5.1 \pm 0.8$ & $5.4 \pm 0.6$ & $0.371^{d}$ \\
\hline
\end{tabular}

${ }^{a}:$ Paired t-test; ${ }^{b}$ : Wilcoxon Rank Test $;^{c}$ : Repeated measures ANOVA;

${ }^{d}$ : Friedman Statistic.

HR, Heart Rate; RPE, Rating of Perceived Exertion.

7 vs. $130 \pm 6, P=0.149$, RPE: $5.1 \pm 0.8$ vs. $5.4 \pm 0.6, P=$ $0.371)$.

\section{Discussion}

It has been shown that the efficacy of CPR significantly affects the chances of survival in a victim with cardiorespiratory arrest [22].

The quality of baseline simulated CPR of these lifeguards is close to the "gold standard" [21] of 70\% quality, however, we have seen in our study and others that performing a high intensity effort (such as an aquatic rescue) can cause significant physiological stress of the lifeguard and that this is associated with a decrease in the quality of CPR [14, 18, 19, 22].

The quality of correct chest compressions was very high (> $90 \%$ ), however, it decreased significantly after performing the 50 meter and 100 meter rescues $(P<0.05)$. Other studies have also shown that after performing an aquatic rescue, the number of correct chest compressions is significantly reduced.

Barcala-Furelos et al. [22] found a significant decrease in the quality of compressions ( $86 \%$ to $66 \%$ ) after performing a rescue of 75 meters in a swimming pool setting. Two years later, the same author also reported a significant decrease in the quality of compressions ( $82 \%$ to $56 \%$ ) after performing a 200 meter rescue with fins on the beach [20]. Finally, AbelairasGómez et al. [19], found a significant decrease in compression quality (from $89 \%$ to $61 \%$ ) after a rescue of 150 meters with fins, also on the beach.

It has been shown that the total number of compressions after an aquatic rescue increases with respect to those performed in a simulated baseline CPR setting [18, 22]. Similarly, in our study, the total number of compressions increased significantly after performing the two rescues with respect to baseline simulated CPR, which caused an exaggerated increase in the rate of compressions leading to a significant decrease in the percentage of compressions with adequate rhythm $(P<0.05)$.

An additional parameter that was significantly reduced following the 50 and 100 meter rescues was the correctness of the hand position. It is not clear why this was observed, because in previous studies this parameter has not analyzed in-depth. However, it has been shown that physical fatigue significantly affects the technical performance [23, 24], and, given that CPR is a technical skill, it could happen that physical fatigue caused by the rescue could affect the concentration of lifeguard at the time of placing the hands on the chest at start the resuscitation techniques.

Performing ventilations effectively is a critical and fundamental element for performing high quality CPR in people who have inhaled water in a drowning incident [4], however, the studied lifeguards did not reach a quality of $50 \%$ in terms of ventilation during the simulated CPR sequence. This result was not surprising, since previous research has also showed 
that ventilation quality provided by attending lifeguards in a CPR setting is often sub-optimal [14, 20].

After the 2 rescues, the quality of the ventilations diminished significantly, consistent to that reported in other work studying lifeguard CPR provision [18, 19]. The decrease in the quality of the ventilations is mainly due to an excessive increase in the amount of insufflated air [14, 25]. It is important to avoid the excessive insufflation of air as it can cause a massive gastric inflation which can lead to further complications [25].

Heart rate (HR) measurement and the subjective rating of perceived exertion (RPE) confirms the high metabolic demand associated with performing an aquatic rescue [7,8]. In our study we observed significant differences in both parameters in the 50 and 100 meter rescues $(P<0.05)$.

The 100-meter rescue lasted practically twice as long as the 50-meter rescue, although that did not cause significant differences in any of the CPR variables measured after the rescues. This can provide relevant information for the lifeguard training, since it seems that what really affects the quality of the CPR is the technical mastery in the resuscitation maneuvers and the intensity applied during the rescue; not so much the distance traveled.

The heart rate after the rescue of 50 meters exceeded $85 \%$ of the theoretical maximum [HRmax $=208.75-(0.73 \times$ age $)]$ [17], and $90 \%$ after the rescue of 100 meters. The intensity generated during the rescue can affect the performance of the $\mathrm{CPR}$, for this reason some authors recommend not surpassing $70 \%$ of the $\mathrm{VO}_{2 \max }$ during the rescue [6]. Other authors, however, argue that a lifeguard should be able to perform quality CPR even under extreme physiological stress/fatigue [14], which further justifies the need for good aerobic and anaerobic fitness in lifeguards, such that they can perform CPR appropriately in a rescue setting [26].

\section{Limitations}

This study aimed to be the first to approach the analysis of the distance traveled in an aquatic rescue and its relationship to the effectiveness of simulated CPR.

One of the main limitations of this study is small sample size. This study was carried out after the completion of a training course to be a professional lifeguard and very few days before the start of the working season on the beaches, so we could only count on the participants of this course for three days. The absence of women in the sample is justified since there were none in that course.

Another limitation is the absence of materials to measure other physiological parameters (oxygen saturation, lactate, etc.) that would allow a more complete analysis of how the fatigue generated in both rescues can affect the performance of CPR.

It would be interesting to use other distances or to carry out rescues in other aquatic spaces, since there are other aspects that can affect the fatigue with which the lifeguard copes with CPR, such as the waves on a beach. In addition, including a larger sample can provide more relevant information on this topic.

\section{Conclusions}

Two different rescues caused a similar decrease in the quality of the simulated CPR. In addition, it has been shown in our study that lifeguards have more consistently perform high quality compressions vs ventilations, which often did not reach $50 \%$ of the required quality. As such, ventilations were the most negatively affected action associated to CPR in the aquatic rescue setting.

We suggest that it is the presence of an aquatic rescue that affects the quality of simulated CPR and not the distance of the rescue. We also argue that CPR training provided to lifeguards should focus more on improving the quality of ventilations (vs. compressions), both of which are essential in the acute management of a drowning victim.

\section{AUTHOR CONTRIBUTIONS}

Brais Ruibal-Lista and Sergio López-García designed the study. Brais Ruibal-Lista collected the data. J. Enrique Moral-García analyzed the data. J. Enrique Moral-García and Sergio López-García analyzed the results and Brais Ruibal-Lista and Sergio López-García drafted the manuscript.

\section{ETHICS APPROVAL AND CONSENT TO PARTICIPATE}

The study was approved by the Ethics and Research Committee of the Universidade da Coruña (CEI-UDC). The research was performed in accordance to the Declaration of Helsinki of 1975 .

\section{ACKNOWLEDGMENT}

We would like to thank all lifeguards involved in this study.

\section{FUNDING}

This research received no specific grant from any funding agency in the public, commercial, or not-for-profit sectors.

\section{CONFLICT OF INTEREST}

The authors declare that there is no conflict of interest regarding the publication of this article.

\section{DATA AVAILABILITY}

The data used to support the findings of this study are available from the corresponding author upon request.

\section{REFERENCES}

[1] World Health Organization (WHO). Global report of drowning. Preventing a leading killer. Bloomberg Philanthropies. Geneva. Switzerland. 2016.

[2] Prieto JA, Nistal P, Méndez D, Abelairas-Gomez C, Barcala-Furelos R. Impact of error self-perception of aerobic capacity in the safety and 
efficacy of the lifeguards. International Journal of Occupational Safety and Ergonomics. 2015; 22: 159-163.

[3] Szpilman D, Soares M. In-water resuscitation-is it worthwhile? Resuscitation. 2004; 63: 25-31.

[4] Szpilman D, Bierens JJLM, Handley AJ, Orlowski JP. Drowning. New England Journal of Medicine. 2012; 366: 2102-2110.

[5] Daniel K, Klauck J. Physiological and biomechanical load parameters in life-saving. Biomechanics and Medicine in Swimming. Swimming Science VI. 1992; 321-325.

[6] Reilly T, Wooler A, Tipton M. Occupational fitness standards for beach lifeguards. Phase 1: the physiological demands of beach lifeguarding. Occupational Medicine. 2006; 56: 6-11.

[7] Saborit JA, Soto Mdel V, Díez VG, Sanclement MA, Hernández PN, Rodríguez JE, et al. Physiological response of beach lifeguards in a rescue simulation with surf. Ergonomics. 2010; 53: 1140-1150.

[8] Prieto-Saborit JA, Egocheaga-Rodríguez J, González-Díez V, Montoliu Sanclement MA, Alameda JC. Determinación de la demanda energética durante un salvamento acuático en playa con y sin material auxiliar. Selección. 2001; 10: 211-220. (In Portuguese)

[9] Salvador A, Penteado R, Lisboa F, Corvino R, Peduzzi E, Caputo F. Physiological and metabolic responses to rescue simulation in surf beach lifeguarding. Journal of Exercise Physiology. 2014; 17: 21-31.

[10] Soar J, Perkins GD, Abbas G, Alfonzo A, Barelli A, Bierens JJ, et al. European Resuscitation Council Guidelines for Resuscitation Section 8. Cardiac arrest in special circumstances: electrolyte abnormalities, poisoning, drowning, accidenta hypothermia, hyperthermia, asthma, anaphylaxis, cardiacsurgery, trauma, pregnancy, electrocution. Resuscitation. 2010; 81: 1400-1433.

[11] Schmidt A, Szpilman D, Berg I, Sempsrott J, Morgan P. A call for the proper action on drowning resuscitation. Resuscitation. 2016; 105: e9e10.

[12] Sanz-Arribas I, Aguado-Gómez R, Martínez-de-Haro V. Influencia de las aletas sobre el tiempo de ejecución en los rescates de víctimas con parada cardiorrespiratoria. Retos. 2017; 31: 133-136. (In Spanish)

[13] Abelairas-Gómez C, Romo-Pérez V, Barcala-Furelos R, Palacios-Aguilar $\mathrm{J}$. Efecto de la fatiga física del socorrista en los primeros minutos de la reanimación cardiopulmonar posrescate acuático. Emergencias. 2013; 25 : 184-190. (In Spanish)

[14] Claesson A, Karlsson T, Thorén A, Herlitz J. Delay and performance of cardiopulmonary resuscitation in surf lifeguards after simulated cardiac arrest due to drowning. The American Journal of Emergency Medicine. 2011; 29: 1044-1050.

[15] Moran K, Webber J. Too much puff, not enough push? Surf lifeguard simulated CPR performance. International Journal of Aquatic Research \& Education. 2013; 7: 13-23.

${ }^{[16]}$ Morgan D, Ozanne-Smith J. Surf lifeguard rescues. Wilderness \&
Environmental Medicine. 2013; 24: 285-290.

[17] Tanaka H, Monahan KD, Seals DR. Age-predicted maximal heart rate revisited. Journal of the American College of Cardiology. 2001; 37: 153156.

[18] Barcala-Furelos R, Abelairas-Gomez C, Romo-Perez V, Palacios-Aguilar J. Effect of physical fatigue on the quality CPR: a water rescue study of lifeguards: physical fatigue and quality CPR in a water rescue. The American Journal of Emergency Medicine. 2013; 31: 473-477.

[19] Abelairas-Gómez C, Barcala-Furelos R, Mecías-Calvo M, Rey-Eiras E, López-García S, Costas-Veiga J, et al. Prehospital emergency medicine at the beach: what is the effect of fins and rescue tubes in lifesaving and cardiopulmonary resuscitation after rescue? Wilderness \& Environmental Medicine. 2017; 28: 176-184.

[20] Barcala-Furelos R, Szpilman D, Palacios-Aguilar J, Costas-Veiga J, Abelairas-Gomez C, Bores-Cerezal A, et al. Assessing the efficacy of rescue equipment in lifeguard resuscitation efforts for drowning. The American Journal of Emergency Medicine. 2016; 34: 480-485.

[21] Perkins GD, Colquhoun M, Simons R. Training manikins. In Colquhoun M, Handley A, Evans TR. (eds.) ABC of resuscitation (pp. 97-101). 5th ed. London: BMJ books. 2004.

[22] Barcala-Furelos R, Abelairas-Gomez C, Queiroga AC, García-Soidán JL. CPR quality reduced due to physical fatigue after a water rescue in a swimming pool. Signa Vitae. 2014; 9: 25-31.

[23] Lyons M, Al-Nakeeb Y, Nevill A. The impact of moderate and high intensity total body fatigue on passing accuracy in expert and novice basketball players. Journal of Sports Science \& Medicine. 2006 5: 215227.

[24] Freitas TT, Calleja-González J, Alarcón F, Alcaraz PE. Acute effects of two different resistance circuit training protocols on performance and perceived exertion in semiprofessional basketball players. Journal of Strength and Conditioning Research. 2016; 30: 407-414.

[25] Wenzel V, Idris AH, Banner MJ, Kubilis PS, Williams JLJ. Influence of tidal volume on the distribution of gas between the lungs and the stomach in the nonintubated patient receiving positive-pressure ventilation. Critical Care Medicine. 1998; 26: 364-368.

[26] USLA. An evidence-based review and report by the United States Lifeguard Standards Coalition. International Journal of Aquatic Research. 2008; 5: 61-129.

How to cite this article: Brais Ruibal-Lista, J. Enrique Moral-García, Sergio López-García. Relationship between rescue distance and the quality of simulated CPR: a pilot study with lifeguards. Signa Vitae. 2021;17(3):137-143. doi:10.22514/sv.2021.044. 